\title{
Aufsatz
}

Christian Götter

\section{Von der militärischen Maßnahme zum politischen Machtmittel}

\section{Die Entwicklung der Wirtschaftsblockade im Ersten Weltkrieg}

DOI 10.1515/mgzs-2016-0069

Zusammenfassung: Die zuerst britische, später alliierte Wirtschaftsblockade Deutschlands im Ersten Weltkrieg galt den Zeitgenossen als entscheidender Beitrag zum Kriegsausgang. Während sie in der angloamerikanischen Forschung einige Aufmerksamkeit erhält, wird sie in der deutschen in letzter Zeit eher stiefmütterlich behandelt. Dieser Aufsatz soll ein Beitrag dazu sein, diese Wirtschaftsblockade bei der Beschäftigung mit dem Ersten Weltkrieg stärker zu berücksichtigen. In ihm argumentiere ich dafür, den Ersten Weltkrieg als einen entscheidenden Katalysator für Veränderungen des Umfangs und der Form der Blockade zu verstehen, der aus einem Mittel der Kriegführung des 19. Jahrhunderts ein politisches Druckmittel des 20. (und 21.) Jahrhunderts gemacht hat.

Schlüsselwörter: Erster Weltkrieg, Wirtschaftsblockade, Strategie

"Blockade, as you have yourself so frequently emphasized, is the key of sea war! «1 So schrieb Admiral of the Fleet Lord (John Arbuthnot) Fisher am 22. Juni 1914 an den Marinehistoriker und wesentlichen Vordenker der britischen Seestrategie Julian Corbett. ${ }^{2}$ Fisher selbst befand sich zu dieser Zeit im Ruhestand, aus dem er allerdings schon wenige Wochen später in den aktiven Dienst zurückgerufen

1 Fisher an Corbett, 22.6.1914, zit. nach Years of Power, 1904-1914. Ed. by Arthur Jacob Marder, Oxford 1956 (= Fear God and Dread Nought. The Correspondence of Admiral of the Fleet Lord Fisher of Kilverstone, 2), S. 507 f., Zitat S. 508.

$2 \mathrm{Zu}$ Corbetts Überlegungen im Kontext der seestrategischen Konzepte Großbritanniens siehe auch Uwe Dirks, Julian S. Corbett und die britische Seekriegführung 1914-1918. In: MGM, 37 (1985), 1, S. 35-50; ferner Stephen Cobb, Preparing for Blockade 1885-1914. Naval Contingency for Economic Warfare, Farnham 2013, bes. S. 85-88, 244f., 255 und S. 259.

Kontakt: Christian Götter, TU Braunschweig, E-Mail: C.Goetter@tu-bs.de 
werden sollte, um die britische Royal Navy als First Sea Lord im Krieg gegen Deutschland zu leiten. Sein erklärtes Ziel war es, den Gegner in der Nordsee und im Mittelmeer einzuschließen und ihm so den Zugang zu den Ozeanen und ihren Handelswegen $\mathrm{zu}$ verwehren. ${ }^{3}$ Die Idee einer Blockade Deutschlands war nichts Neues. Ganz im Gegenteil: Blockaden waren die etablierte Vorgehensweise der britischen Seemacht und seit dem Ersten Englisch-Niederländischen Krieg 1653 immer wieder angewendet worden. ${ }^{4}$ Die konkrete, gegen Deutschland angewendete Form war seit den 1880er Jahren entwickelt worden. ${ }^{5}$ Bereits 1906, in seiner ersten Amtszeit als First Sea Lord, hatte Fisher sie als »Our great special antiGerman weapon of smashing an enemy’s commerce « ${ }^{6}$ bezeichnet und ausgeführt:

»It's so very peculiar that Providence has arranged England as a sort of huge breakwater against German commerce, which must all come either one side of the breakwater through Straits of Dover, or the other side of the breakwater the north of Scotland. It's a unique position of advantage that we possess, and such is our naval superiority that on the day of war we smop up 800 German merchant steamers. Fancy the `knock-down` blow to German trade and finance! Worth Paris! ${ }^{7}$

Freilich ging es ihm nicht allein um wirtschaftliche Ziele. Er wollte mit der Blockade eine Seeschlacht zwischen den gewaltigen Schlachtflotten beider Seiten provozieren, diese gewinnen und Großbritanniens Seemacht erneut so klar demonstrieren, wie es seinem Idol Horatio Nelson gegen die Marine Napoleon Bonapartes gelungen war. Wäre dies vollbracht, könnte eine britische Armee landen, wo immer es wünschenswert sei - beispielsweise an der Ostseeküste, vor den Toren Berlins. ${ }^{8}$

3 Fisher an Corbett, 22.6.1914, zit. nach Years of Power (wie Anm. 1), S. 507 f.

4 Eric J. Grove, Blockade. In: Actium. Battle of - Ex Voto. Ed. by John B. Hattendorf, Oxford 2007 (= The Oxford Encyclopedia of Maritime History, 1), S. 298-303, hier S. 298.

5 Siehe vor allem Julian S. Corbett, Some Principles of Maritime Strategy, Annapolis, MD 1988 (Original 1911), S. 183-196, 208; sowie Cobb, Preparing for Blockade (wie Anm. 2); Shawn T. Grimes, Strategy and War Planning in the British Navy, 1887-1918, Woodbridge 2012; Matthew S. Seligmann, The Royal Navy and the German Threat, 1901-1914. Admiralty Plans to Protect British Trade in a War Against Germany, Oxford 2012; ferner Nicholas A. Lambert, Planning Armageddon. British Economic Warfare and the First World War, Cambridge, MA 2012; die quellenreiche Studie von Eric W. Osborne, Britain’s Economic Blockade of Germany 1914-1919, London 2004; Bruce Russell, Prize Courts and U-boats. International Law at Sea and Economic Warfare during the First World War, Dordrecht 2009, S. 41-60.

6 Fisher an Captain Seymour Fortescue, Equerry in Waiting to King Edward VII 1893-1910, 14.4.1906, zit. nach Years of Power (wie Anm. 1), S. 71f., Zitat S. 72.

7 Ebd.

8 Fisher an Esher, 20.9.1911, zit. nach Years of Power (wie Anm. 1), S. 383-386. Corbett hatte sowohl die wirtschaftliche als auch die militärische Ebene einer Blockade ausführlich diskutiert, beachte Corbett, Some Principles (wie Anm. 5), S. 65-71 und S. 102. 
Verwirklicht wurden diese Pläne im Ersten Weltkrieg nicht. Obwohl durchaus versucht wurde, die britische Seemacht für Flankenangriffe auf die Kontinentalmächte Deutschlands, Österreich-Ungarns und des Osmanischen Reiches zu nutzen, ${ }^{9}$ blieb die britische Armee im Wesentlichen an der Seite von Frankreich an der Westfront konzentriert. Ein Teil der Pläne Fishers wurde jedoch umgesetzt: Mit Kriegsbeginn wurde eine Blockade Deutschlands errichtet. Zwar wurde sie, um die mit dem Begriff verbundenen rechtlichen Folgen $\mathrm{zu}$ umgehen, nicht offiziell so genannt, praktisch aber wurde sie etabliert und blieb über den Waffenstillstand hinaus bis 1919 bestehen. Sie bildete ein wichtiges Druckmittel während der Friedensverhandlungen, ${ }^{10}$ auch wenn sie auf Drängen der US-Regierung ab Ende 1918 entspannt wurde. ${ }^{11}$ Ihre Wirkung auf den Kriegsverlauf galt den an ihr beteiligten Zeitgenossen als enorm. Der First Lord of the Admiralty (Marineminister) Winston Churchill sah die Zeit als Großbritanniens Verbündeten. ${ }^{12}$ Der erste Chef des 10. Kreuzergeschwaders, das im Norden Großbritanniens die Blockade überwachte, der ehemalige Naval Secretary des First Lord of the Admiralty, Admiral Sir Dudley de Chair, sah die Blockade als Kern der britischen Strategie, Deutschland mithilfe von Seemacht $\mathrm{zu}$ bezwingen. Er betonte, dass sie ihre Wirkung auf den deutschen Handel schon zu entfalten begann, bevor sie vollständig errichtet war. ${ }^{13}$ Sein Nachfolger, Admiral Sir Reginald Tupper, war überzeugt, dass »the stranglehold of the blockade made it possible for the Allies to vanquish Germany. «14 Admiral Sir David Beatty, der Befehlshaber der britischen Grand Fleet in der zweiten Kriegshälfte, meinte Anfang 1917 gegenüber seiner Frau:

9 Namentlich an den Dardanellen, wo um die Kontrolle über den Bosporus gekämpft wurde, die es den Mittelmächten zusammen mit der Herrschaft über die Ostsee erlaubte, die Verbindungen zwischen den westlichen Alliierten und Russland zu blockieren, Paul G. Halpern, A Naval History of World War I, London 1994, S. 101-110.

10 Beachte Winston Churchills Stellungnahme, Number of Land Forces. In: Hansard, House of Commons Debates, Bd 113, 3.3.1919, Sp. 69-183, hier Sp. 84; Avner Offer, The First World War. An Agrarian Interpretation, Oxford 1989, S. 2, 6, 383f. und S. 386.

11 Archibald Colquhoun Bell, A History of the Blockade of Germany and of the Countries Associated with Her in the Great War: Austria-Hungary, Bulgaria, and Turkey 1914-1918, London 1937, S. 705-709; Paul Halpern, World War I: The Blockade. In: Naval Blockades and Seapower. Strategies and Counter-Strategies, 1805-2005. Ed. by Bruce A. Elleman and S.C.M. Paine, Abingdon 2006, S. 91-103, hier S. 96f.

12 Siehe Alan Kramer, Blockade and Economic Warfare. In: The State. Ed. by Jay Winter, Cambridge 2014 (= The Cambridge History of The First World War, 2), S. 460-489, hier S. 465.

13 Admiral Sir Dudley de Chair, The Sea is Strong. Ed. by Somerset de Chair, London 1961, S. 169 und S. 176f.; beachte auch Osborne, Britain's Economic Blockade (wie Anm. 5), S. 61.

14 Admiral Sir Reginald Tupper, Reminiscences, London 1929, S. 217; siehe auch ebd., S. 264. 
»[T]he blockade [...] is most effective, and indeed it is the only thing that is winning the war for us. Our armies cannot advance, but slowly \& surely we are strangling them with my old 10th Cruiser Squadron. They are doing wonderful work, no gale is too much for them and they stop 95 per cent of the ships passing through, and the weather has been pretty bad. $\ll^{15}$

Der langjährige Sekretär des Committee of Imperial Defence, des strategischen Beratergremiums der britischen Regierung, Maurice Hankey, war nach dem Krieg der Ansicht, dass die Blockade »if not sthe decisive factor`, at least >ne of the decisive factors in the victory of the Allied and Associated Powers « gewesen sei. ${ }^{16}$ Auch einer der ersten Historiker der Blockade, Keble Chatterton, meinte, sie sei dafür verantwortlich gewesen, »that our enemy finally collapsed. $\aleph^{17}$ Sogar der Nachkriegspremierminister Andrew Bonar Law habe in ihr den Grund für den Zusammenbruch Deutschlands gesehen. ${ }^{18}$ Archibald Bell, der die "offizielle Geschichte « der Blockade für das Foreign Office verfasste, widmete ihrer Wirkung ein eigenes Kapitel. ${ }^{19}$ Auf deutscher Seite wurden schon vor dem Krieg in Marinekreisen »Siechtum und Elend « infolge einer Blockade befürchtet. ${ }^{20}$ Später führten beispielsweise Großadmiral Alfred von Tirpitz und General Erich Ludendorff die Blockade als Ursache deutscher Probleme im Kriegsverlauf an. Letzterer sah in durch sie ausgelösten wirtschaftlichen Zwängen gar Ursachen für strategische Entscheidungen der dritten Obersten Heeresleitung. ${ }^{21}$ Generell herrschte in der Zwischenkriegszeit in Deutschland wie in Großbritannien die Ansicht vor, dass die britische Blockade wesentlich zur deutschen Niederlage beigetragen habe.22 Deshalb bereitete sich Großbritannien darauf vor, auch in einem künftigen

15 Beatty an seine Frau, 5.2.1917, zit. nach 1902-1918. Ed. by M. A. Ranft, Aldershot 1989 (= The Beatty Papers. Selections from the Private and Official Correspondence of Admiral of the Fleet Earl Beatty, 1), S. 399f.

16 Maurice Pascal Alers Hankey, The Supreme Command, 1914-1918, 2 vols., London 1961, hier vol. 2, S. 859.

17 Edward Keble Chatterton, The Big Blockade, London 1931, S. 28.

18 Ebd., S. V.

19 Bell, A History of the Blockade (wie Anm. 11), S. 671-692.

20 Die Bedeutung des Außenhandels für unsere Volkswirtschaft. In: 1914. Hrsg. von Nauticus, Berlin 1914 (= Jahrbuch für Deutschlands Seeinteressen, 16), S. 311-363, hier S. 360.

21 Alfred von Tirpitz, Erinnerungen, 2. Aufl., Leipzig 1920, S. 313; Erich Ludendorff, Kriegführung und Politik, 2. Aufl., Berlin 1922, S. 157 f.; beachte auch Erich Raeder, Mein Leben. Oberbefehlshaber der deutschen Kriegsmarine 1935-1943, Beltheim-Schnellbach 2009, S. 222; August Skalweit, Die deutsche Kriegsernährungswirtschaft, Stuttgart 1927, S. 1f. und S. $23-25$.

22 Geoffrey Till, Naval Blockade and Economic Warfare in the European War, 1939-45. In: Naval Blockades (wie Anm. 11), S. 117-130, hier S. 117f.; beachte für Deutschland speziell die Übersicht bei Anne Roerkohl, Hungerblockade und Heimatfront. Die kommunale Lebensmittelversorgung in Westfalen während des Ersten Weltkrieges, Stuttgart 1991, S. 15-18. 
Krieg gegen Deutschland auf diese Maßnahme zurückzugreifen, und installierte zu Beginn des Zweiten Weltkriegs eine Kombination aus maritimen und bürokratischen Maßnahmen, die denen des Ersten Weltkriegs stark ähnelten. ${ }^{23}$ Das nationalsozialistische Deutschland auf der anderen Seite war nicht zuletzt im Rahmen seiner Autarkiepolitik bestrebt, sich gegen eine solche Wiederholung abzusichern. ${ }^{24}$

Insbesondere nach dem Zweiten Weltkrieg jedoch wurde die Blockade kritischer betrachtet. Einer der wichtigsten Historiker der Marinegeschichte im Ersten Weltkrieg, Paul G. Halpern, gab zu bedenken, dass die tatsächliche Wirkung der Blockade kaum gemessen werden könne, ${ }^{25}$ und räumte ihr in seinem Standardwerk »A Naval History of World War I« nur fünf Seiten ein. ${ }^{26}$ Alan Kramer wies darauf hin, dass mit Blick auf die wirtschaftliche Seite des Krieges die Auswirkungen der Blockade durch den Umstand in den Schatten gestellt wurden, dass die Mittelmächte einen Krieg gegen ihre wichtigsten Handelspartner führten, wobei letztere die Mehrzahl kriegswichtiger Rohstoffe kontrollierten. ${ }^{27}$ Insbesondere in der deutschen Geschichtswissenschaft führte die Blockade letzthin geradezu ein Schattendasein. Anne Roerkohl meinte, eine weniger fatale Nahrungsmittelverwaltung in Deutschland hätte die durch sie hervorgerufenen Ausfälle kompensieren können. ${ }^{28}$ Sönke Neitzel betonte, dass sie die Nahrungsproblematik in Deutschland zwar verschärft, aber keineswegs ausgelöst habe. ${ }^{29}$ Ein eigenes Stichwort im wichtigsten deutschsprachigen Nachschlagewerk zum

23 Grove, Blockade (wie Anm. 4), S. 302; Robert W. Matson, Neutrality and Navycerts. Britain, the United States, and Economic Warfare, 1939-1940, New York 1994, S. $19 \mathrm{f}$.

24 Siehe David E. Kaiser, Economic Diplomacy and the Origins of the Second World War. Germany, Britain, France, and Eastern Europe, 1930-1939, Princeton, NJ 1980; Rolf-Dieter Müller, Totaler Krieg und Wirtschaftsordnung. Ausnahmezustand oder Chance eines grundlegenden Wandels? Deutsche Experimente in zwei Weltkriegen. In: Erster Weltkrieg - Zweiter Weltkrieg. Ein Vergleich. Krieg, Kriegserlebnis, Kriegserfahrung in Deutschland. Hrsg. von Bruno Thoß und Hans-Erich Volkmann, Paderborn [u. a.] 2002, S. 43-55, hier S. 48; zur entsprechenden Tradition seit dem ausgehenden 19. Jahrhundert siehe Offer, The First World War (wie Anm. 10), S. 324; zu Überlegungen im Ersten Weltkrieg Albrecht Ritschl, The Pity of Peace. Germany's Economy at War, 1914-1918 and Beyond. In: The Economics of World War I. Ed. by Stephen Broadberry and Mark Harrison, Cambridge 2005, S. 41-76, hier S. 63f.

25 Halpern, World War I (wie Anm. 11), S. 103.

26 Halpern, A Naval History (wie Anm. 9), S. 48-50 und S. 291 f.

27 Kramer, Blockade (wie Anm. 12).

28 Roerkohl, Hungerblockade (wie Anm. 22), S. 317; beachte ebenfalls Stephen Broadberry and Mark Harrison, The Economics of World War I. An Overview. In: The Economics of World War I (wie Anm. 24), S. 3-40, hier S. 19.

29 Sönke Neitzel, Von Wirtschaftskriegen und der Wirtschaft im Kriege. In: Krieg und Wirtschaft. Von der Antike bis ins 21. Jahrhundert. Hrsg. von Wolfram Dornik [u. a.], Innsbruck 2010, 
Ersten Weltkrieg, der »Enzyklopädie Erster Weltkrieg«, erhielt sie nicht und Michael Salewski vertrat die Ansicht, dass der Seekrieg sich auf den Ausgang des Ersten Weltkriegs insgesamt kaum ausgewirkt habe - was entsprechend auch für die Blockade gelte. ${ }^{30}$ Gerade Untersuchungen angloamerikanischer Historiker kamen jedoch auch wieder zu anderen Urteilen. Schon Avner Offer hatte darauf hingewiesen, dass die Blockade eine wichtige Komponente des Krieges gegen Deutschland gewesen war und während des Waffenstillstands entscheidend wurde. ${ }^{31}$ Bruce Russell betonte den Rückgang des deutschen Außenhandelsvolumens im Kriegsverlauf. ${ }^{32}$ Eric W. Osborne sah in der Blockade den wichtigsten Grund für den »Steckrübenwinter« 1916/17 und darüber hinaus für den Sieg der alliierten und assoziierten Mächte im Ersten Weltkrieg, wobei seine Argumentation nicht völlig überzeugt. ${ }^{33}$ Nicholas A. Lambert schließlich erkannte im britischen Konzept des Wirtschaftskriegs gar »a national strategy of quick, decisive war comparable in function and objectives to Germany's infamous Schlieffen Plan. ${ }^{34}$ Auch an dieser These bleiben Zweifel. ${ }^{35}$ John D. Grainger merkte bereits zusammenfassend an, dass die Wirkung der Blockade gemeinhin von ihren Kritikern unter-, von ihren Befürwortern allerdings überbewertet werde. ${ }^{36}$ Er jedoch stellt kontrafaktisch die Frage, ob nicht die Kaiserliche Marine ohne die britische Blockade auf den U-Boot-Krieg verzichtet hätte, was möglicherweise den Kriegseintritt der USA hätte verhindern können. ${ }^{37}$ Eine einheitliche Forschungsmeinung zur britischen Blockade im Ersten Weltkrieg existiert also nicht. Unabhängig davon lässt sich allerdings festhalten, dass in Deutschland wie Großbritannien von den Zeitgenossen an den Erfolg der britischen Blockade geglaubt wurde - und dass an deren prinzipielles Potenzial bis heute geglaubt

S. 49-66, hier S. 57. Grimes, Strategy (wie Anm. 5), S. 191f., sieht eine ähnliche Kombination verschiedener Faktoren, spricht der Blockade aber einen größeren Anteil zu.

30 Michael Salewski, Seekrieg. In: Enzyklopädie Erster Weltkrieg. Hrsg. von Gerhard Hirschfeld, Gerd Krumeich und Irina Renz, Paderborn [u.a.] 2003, S. 828-832. Am Rande berührt wird die Blockade beispielsweise immerhin bei Gustavo Corni, Ernährung. In: ebd., S. 461-464; HansPeter Ullmann, Kriegswirtschaft. In: ebd., S. 220-232. Zum Schattendasein, das die Blockade in allgemeinen Werken führt, siehe auch Cobb, Preparing for Blockade (wie Anm. 2), S. $259 \mathrm{f}$.

31 Offer, The First World War (wie Anm. 10), S. 68, 76-78 und S. 386.

32 Russell, Prize Courts (wie Anm. 5), S. 134-136.

33 Osborne, Britain's Economic Blockade (wie Anm. 5), S. 1-4 und S. 144; beachte auch Nigel Hawkins, The Starvation Blockades, Barnsley 2002, S. 236-239.

34 Lambert, Planning Armageddon (wie Anm. 5), S. 1.

35 Beachte beispielsweise Kramer, Blockade (wie Anm. 12), S. 463.

36 John D. Grainger, Introduction. In: The Maritime Blockade of Germany in the Great War. The Northern Patrol, 1914-1918. Ed. by John D. Grainger, Aldershot 2003, S. 1-24, hier S. 22.

37 Ebd., S. 18. 
wird. ${ }^{38}$ Wirtschaftliche Druckmittel erfreuten sich nach dem Ersten Weltkrieg über das 20. Jahrhundert hinweg sogar zunehmender Beliebtheit und gelten auch im frühen 21. Jahrhundert als ein wirksamer Weg, um Außenpolitik mit anderen Mitteln fortzusetzen - ohne dafür Kriege führen zu müssen. ${ }^{39}$ Sie werden nach wie vor oftmals nicht als Blockaden bezeichnet, um die rechtlichen Konsequenzen zu umgehen, die mit diesem Begriff verbunden sind. ${ }^{40}$

Im Folgenden soll nicht nach den schwerlich genau zu beziffernden Auswirkungen der britischen Blockade Deutschlands auf den Ersten Weltkrieg gefragt werden. Vielmehr geht es darum, die Perspektive zu wechseln und nach den Auswirkungen des Ersten Weltkriegs auf Form und Methode der Blockade zu fragen. Diese nämlich waren, was in der bisherigen Forschung kaum in den Fokus genommen wird, gewaltig. Denn im Verlauf des Ersten Weltkriegs wurde die Blockade transformiert von einem Mittel der Kriegführung des 19. Jahrhunderts hin zu einem politischen Druckmittel für das 20. und 21. Jahrhundert. ${ }^{41}$ Der Erste Weltkrieg, so die These dieses Aufsatzes, war für die Evolution des Konzepts der Blockade ein entscheidender Katalysator; eine Eigenschaft, die der Konflikt auch im Hinblick auf andere Felder hatte - was durch die Konzentration auf seine Rolle als »Urkatastrophe« oftmals weniger beachtet wird. Doch zurück zur Blockade: Sowohl ihre Gestalt als auch die Art ihrer Umsetzung wurden im Verlauf des Ersten Weltkriegs wesentlich verändert und ließen sie über die kriegsspezifischen rechtlichen Schranken hinauswachsen, die ihr im Verlauf des 19. Jahrhunderts gegeben worden waren. ${ }^{42}$ Beide Veränderungen waren einerseits eine Folge der

38 Beachte Geoffrey Till, Series Editor's Preface. In: Osborne, Britain's Economic Blockade (wie Anm. 5), S. VIIf.; dagegen Tor E. Forland, The History of Economic Warfare: International Law, Effectiveness, Strategies. In: Journal of Peace Research, 30 (1993), 2, S. 151-162, hier S. 160.

39 Beachte Wolff Heintschel von Heinegg, Naval Blockade and International Law. In: Naval Blockades (wie Anm. 11), S. 10-22, hier S. 10; Economic Sanctions Reconsidered. Ed. by Gary Clyde Hufbauer [u.a.], 3. ed., Washington, DC 2007, S. 10; R.T. Naylor, Economic Warfare. Sanctions, Embargo Busting, and Their Human Cost, Boston 1999; International Sanctions. Between Words and Wars in the Global System. Ed. by Peter Wallensteen and Carina Staibano, London 2005.

40 Bruce A. Elleman and S.C.M. Paine, Introduction. In: Naval Blockades (wie Anm. 11), S. 3-9, hier S. 6.

41 Vergleiche Chatterton, The Big Blockade (wie Anm. 17), S. 11. Zur Rechtspraxis, die nach wie vor weitgehend auf den Prinzipien der Erklärung von London und der Praxis der Weltkriege fußt, sieht man von humanitären und technologischen Anpassungen sowie einigen Auslegungskonflikten ab, siehe Heintschel von Heinegg, Naval Blockade (wie Anm. 39), besonders S. 13-20.

42 Forland, The History of Economic Warfare (wie Anm. 38), S. 152f. Sie wurde somit gewissermaßen wieder dem politischen Druckmittel ähnlicher, das sie in den napoleonischen Kriegen gewesen war, beachte Elleman/Paine, Introduction (wie Anm. 40), S. 4f.; Silvia Marzagalli, 
Erfahrungen, die im Kriegsverlauf mit der Blockade gemacht wurden und die durch theoretische Überlegungen außerhalb eines Krieges nicht $\mathrm{zu}$ ersetzen gewesen wären, und andererseits eine Folge der Eskalation des Ersten Weltkriegs, der sich mehr und mehr totalisierte und so Grenzüberschreitungen möglich machte, die anders nicht denkbar gewesen wären. Wie diese Transformation eines relativ klar umrissenen Mittels der Kriegführung $\mathrm{zu}$ einem weitgehend schrankenlosen politischen Druckmittel vonstatten ging, soll hier in zwei Schritten demonstriert werden. Zunächst wird gezeigt, wie die Blockade verschärft wurde, sowohl inhaltlich als auch hinsichtlich ihrer Umsetzung. Anschließend wird verdeutlicht, wie die Durchsetzung der Blockade von der hohen See auf die Schreibtische von Mitarbeitern der Blockadeorganisationen verlegt wurde. In beiden Schritten soll so die Bedeutung des Ersten Weltkriegs als Katalysator der Veränderungen herausgearbeitet werden. Dabei steht die britische Seite im Fokus, während die deutsche Sicht nur am Rande berücksichtigt wird. Für diese Konzentration sprechen zwei Gründe. Hinsichtlich des Umfangs und der Schärfe der Blockade brächte die deutsche Perspektive allenfalls eine Ergänzung der am britischen Beispiel deutlich werdenden Veränderungen mit sich. ${ }^{43}$ Für die Analyse der methodischen Verlagerung von einer militärischen zur politischdiplomatischen Maßnahme liefert sie keine neuen Erkenntnisse, da die praktische Umsetzung des deutschen Handelskrieges zur See den gesamten Krieg über als militärische Maßnahme verfolgt wurde.

\section{Von Waffen zu Peperoni - Wachsender Umfang und zunehmende Schärfe der Blockade}

$\mathrm{Zu}$ Kriegsbeginn waren Art und Anzahl der blockierten Güter vergleichsweise überschaubar und an der »Erklärung von London « ausgerichtet. ${ }^{44}$ Diese Übereinkunft über Seekrieg und Blockaden war 1909 von den Großmächten in London verabschiedet, jedoch nie ratifiziert worden, nachdem sie im britischen Oberhaus gescheitert war. Dennoch stellte sie so etwas wie eine gemeinsame Basis dar, wenn es darum ging, die Aktionen der am Krieg beteiligten Staaten auf der internationalen Bühne zu rechtfertigen, vor allem vor den USA, einem der wich-

Napoleon's Continental Blockade. An Effective Substitute to Naval Weakness? In: Naval Blockades (wie Anm. 11), S. 24-33, hier S. 25.

43 Beachte hierzu insbesondere Hawkins, The Starvation Blockades (wie Anm. 33).

44 Das galt auch für Deutschlands Haltung, siehe Cobb, Preparing for Blockade (wie Anm. 2), S. 252. 
tigsten Handelspartner beider Seiten bei Kriegsbeginn. ${ }^{45}$ Die britische Admiralty gab sie gegenüber ihren Offizieren bei Kriegsbeginn sogar als bindende Regel an. ${ }^{46}$ Die von Großbritannien am 4. August 1914 proklamierte Liste der »absoluten Konterbande«, also der Waren, die beschlagnahmt werden sollten, wann immer sie für den Feind bestimmt waren, bestand aus zwölf Punkten. ${ }^{47}$ Diese umfassten alle Arten von Waffen und Geschossen sowie deren jeweilige Bestandteile; für den Krieg produzierte Sprengstoffe, Geschütze, militärische Transportmittel, militärische Ausrüstung und Kleidung; Lasttiere, die im Krieg eingesetzt werden konnten; Ausrüstung für Feldlager, Panzerung, Kriegsschiffe, Flugzeuge, Luftschiffe, Ballone und ihre Komponenten sowie Maschinen, die der Produktion oder Reparatur von Waffen oder Munition dienten. ${ }^{48}$ Die Liste entsprach der Erklärung von London, sieht man davon ab, dass ihr Luftfahrzeuge hinzugefügt worden waren, wozu Kriegsteilnehmer durchaus ermächtigt waren. ${ }^{49}$ Daneben existierte eine 13 Einträge umfassende Liste bedingter Konterbande; solcher Waren also, die beschlagnahmt werden konnten, »if it is shown to be destined for the use of the armed forces or of a government department of the enemy State«, es sei denn, wenn es offensichtlich war, dass die Waren für den Krieg nicht nützlich waren oder wenn das Schiff keinen vom Gegner kontrollierten Hafen ansteuerte. ${ }^{50}$ Bedingte Konterbande, zu denen nicht zuletzt Nahrung und Futtermittel für Tiere gehörten, konnten also zunächst nicht beschlagnahmt werden, wenn sie für einen neutralen Hafen bestimmt waren, selbst wenn sie dann über den Landweg an den Kriegsgegner weiterverkauft wurden. ${ }^{51}$ Am Beispiel dieses Prinzips der sogenannten continuous voyage, ${ }^{52}$ insbesondere im Hinblick auf Nahrungsmittel, lässt sich die gesteigerte Schärfe der Blockaderegeln angesichts der Kriegserfahrungen sehr gut ablesen.

45 Beachte die Einschätzung bei Ottmar Bühler, Neutralität, Blockade und U-Boot-Krieg in der Entwicklung des modernen Völkerrechts, Berlin 1940, S. 25 f.

46 Cobb, Preparing for Blockade (wie Anm. 2) S. 75f.; siehe auch Chair, The Sea is Strong (wie Anm. 13), S. 185.

47 Erklärung von London, Artikel 22 und 30. Die Erklärung findet sich online beispielsweise unter <www.icrc.org/ihl/INTRO/255?OpenDocument> (letzter Zugriff 8.6.2016).

48 Königliche Proklamation der Konterbande, 4.8.1914, London Gazette, 5.8.1914, Supplement, S. $6163 \mathrm{f}$.

49 Erklärung von London (wie Anm. 47), Artikel 23 und 25.

50 Siehe Artikel 33-35 der Erklärung von London (wie Anm. 47).

51 Das entsprach der Erklärung von London (wie Anm. 47); siehe auch Chair, The Sea is Strong (wie Anm. 13), S. 185.

52 Für eine gut verständliche, zeitgenössische Auseinandersetzung beachte Charles Burke Elliott, The Doctrine of Continuous Voyages. In: The American Journal of International Law, 1 (1907), 1, S. 61-104, bes. S. 67. 
Bereits am 20. August verschärfte die britische Regierung nämlich das eigene Vorgehen. Sie verwies darauf, dass Russland und Frankreich erklärt hätten, sich im Wesentlichen an die Erklärung von London halten $\mathrm{zu}$ wollen, und gab an, sich dieser Haltung um der Einheitlichkeit des Vorgehens willen anzuschließen. Geradezu lapidar wurden allerdings sechs Modifikationen verkündet, die gegenüber der ursprünglichen Erklärung vorgenommen würden. Drei dieser Veränderungen waren von größerer Bedeutung. So behielt sich Großbritannien vor, neutrale Schiffe auch noch auf ihrer Rückreise zu beschlagnahmen, wenn diese mithilfe falscher Papiere Konterbande an den Gegner geliefert hatten. Zudem beanspruchte die britische Regierung das Recht, jegliche denkbaren Beweise heranzuziehen, um das wirkliche Ziel der Waren neutraler Händler festzustellen, nicht allein die in der Erklärung von London vorgesehenen Schiffspapiere. Ferner erklärte Großbritannien, dass es bedingte Konterbande, die für den Gegner bestimmt seien, immer beschlagnahmen würde - und nicht nur dann, wenn sie auch in einen gegnerischen Hafen geliefert würden. ${ }^{53}$ Damit wurde das Prinzip der continuous voyage für bedingte Konterbande eingeführt, namentlich also auch für Nahrungsmittel. ${ }^{54}$ Zudem wurde eine Drohkulisse für mögliche Blockadebrecher aufgebaut: Sie liefen Gefahr, ihre Schiffe zu verlieren, zumindest aber durch Prüfungen in britischen Häfen Zeit zu verlieren und somit wirtschaftliche Verluste zu machen. Abgesehen von der Einheitlichkeit des alliierten Vorgehens wurde diese Ausweitung der Blockade dadurch gerechtfertigt, dass die Admiralty erfahren zu haben glaubte, in Deutschland würden Nahrungsmittel zentral vom Staat verteilt, was innerhalb der bestehenden Regelwerke ein legitimer Grund gewesen wäre, sich aber bald als Irrtum herausstellte. ${ }^{55}$ Allerdings hatte die britische Regierung zusätzlich den Umstand, dass Deutschland am 5. August Minen in internationalen Gewässern vor Suffolk gelegt habe, als Verletzung der internationalen Seekriegsregeln gewertet und damit eigene Schritte in dieser Richtung gerechtfertigt. ${ }^{56}$

Für die Blockade war diese Ausweitung von großer Bedeutung. Mit ihr ging die britische Regierung das Problem an, dass Deutschland Nachschub aus Übersee auch indirekt über seine neutralen Nachbarländer importieren konnte. Dass dieses Problem bestehen würde, war bereits vor dem Krieg diskutiert wor-

53 Proklamation, 20.8.1914, London Gazette, 22.8.1914, Supplement, S. 673 f.

54 Cobb, Preparing for Blockade (wie Anm. 2), S. 76 und S. 252; Chatterton, The Big Blockade (wie Anm. 17), S. 29-31; Osborne, Britain's Economic Blockade (wie Anm. 5), S. 63.

55 Cobb, Preparing for Blockade (wie Anm. 2), S. 252; Osborne, Britain's Economic Blockade (wie Anm. 5), S. $64 \mathrm{f}$.

56 Hankey, The Supreme Command, vol. 1 (wie Anm. 16), S. 13f.; Osborne, Britain's Economic Blockade (wie Anm. 5), S. 62; beachte ferner Chatterton, The Big Blockade (wie Anm. 17), S. 29-31. 
den. David Lloyd George hatte im Herbst 1912 darauf hingewiesen, dass jegliche Versuche, Deutschland vom internationalen Seehandel abzuschneiden, scheitern würden, wenn Belgien und die Niederlande neutral blieben und frei handeln könnten. Er hatte damals die Ansicht vertreten, dass man diesen Ländern im Fall des Falles nur die Importe gestatten dürfe, die ihrem eigenen Verbrauch entsprachen. Der First Lord der Admiralty, Winston Churchill, hatte derlei Kritik jedoch ignoriert, indem er die Neutralität dieser Staaten im Kriegsfall für unmöglich erklärte. ${ }^{57}$ Mit der Einführung der continuous voyage für bedingte Konterbande schien das Problem nun gelöst. Spätere Behauptungen, die Erklärung von London ebenso wie die früheren Abkommen zum Seekriegsrecht in Den Haag und die Erklärung von Paris von 1856 hätten der britischen Blockade unerträgliche Schranken auferlegt und verhindert, dass sie eine schnelle(re) Kriegsentscheidung hätte bringen können, wie sie beispielsweise Hankey erhob, lassen sich angesichts der Blockade in der Fassung vom 20. August nicht bestätigen. Großbritannien hatte sich über diejenigen Teile der Vereinbarungen, die seine geplante Strategie hätten behindern können, mit einem Federstrich hinweggesetzt.

Auch dass die Listen der absoluten und bedingten Konterbande weitgehend den Entwürfen aus der Erklärung von London entsprachen, lässt sich nicht als Beleg dafür heranziehen, dass diese Erklärung die Effektivität der Blockade beeinträchtigt hätte. Denn zum einen gab es schlicht keine anderen Listen diejenigen, die Großbritannien verkündete, waren vielmehr das Ergebnis einer Expertenkommission, des Trading with the Enemy Committee, das sich ab 1911 mit Detailfragen der Wirtschaftskriegführung befasst hatte. ${ }^{58}$ Sicherlich fußten sie auf den Listen der Erklärung von London - waren doch diese 1909 von eben solchen Experten aus den verschiedenen Staaten der Welt als Konsens zusammengetragen worden, also auch von den britischen Fachleuten. Sie waren dennoch, cum grano salis, der Stand der Dinge. Zum anderen ließ sich Großbritannien, wie bereits gezeigt, durch die existierenden Listen nicht beschränken, denn schon in der ursprünglichen Fassung der eigenen Konterbandelisten waren Luftfahrzeuge von bedingter $\mathrm{zu}$ absoluter Konterbande aufgewertet worden. Die britische Regierung ließ sich von der Erklärung also sicherlich leiten, allerdings in keiner Weise gängeln.

Dass die Schärfe der Blockade wenige Wochen später wieder reduziert wurde, entsprang ebenfalls nicht einem häufig laut beklagten Bedürfnis des Foreign Office, sich an die im Frieden ausgehandelten Verträge zu halten; es war eine

57 Osborne, Britain's Economic Blockade (wie Anm. 5), S. 54-56; The National Archives (TNA), Cab 38/22/42, Protokoll der 120. Sitzung des Committee of Imperial Defence vom 6.12.1912, S. 7.

58 Hankey, The Supreme Command, vol. 1 (wie Anm. 16), S. 90-92; zum Aufbau dieser Kommissionen siehe ebd., S. 85-89. 
Folge nicht zuletzt diplomatischen Drucks. ${ }^{59}$ Die europäischen Neutralen bekamen durch das britische Vorgehen selbst Probleme mit ihrer Versorgung. ${ }^{60}$ Auch die USA protestierten. ${ }^{61}$ Am 29 . Oktober 1914 wurde daher die Regel der continuous voyage für bedingte Konterbande wieder eingeschränkt: Es sollte nur noch dann beschlagnahmt werden, wenn der Empfänger im neutralen Hafen nicht ausgezeichnet, ein feindlicher Agent oder die Ware »to order« bestimmt sei. ${ }^{62}$ Die Blockade wurde damit also für Stoffe, die nicht zu absoluter Konterbande erklärt werden konnten, abgeschwächt, »in order to minimise, so far as possible, the interference with innocent neutral trade occasioned by the war «. ${ }^{63}$ Relevant war dies vor allem für die ersten Artikel auf der Liste der bedingten Konterbande, Nahrung und Tierfutter. ${ }^{64}$ Allerdings bedeutete der Verzicht auf die continuous voyage für die bedingte Konterbande nicht, dass Großbritannien nun tatenlos der Lieferung von Nahrungsmitteln an Deutschland zugesehen hätte. Zum einen wurde die Einschränkung später wieder aufgehoben, ${ }^{65}$ zum anderen begann Großbritannien sofort, andere Wege zu suchen, dem Gegner Nahrungsmittel vorzuenthalten; hierauf wird im zweiten Abschnitt wieder Bezug genommen.

Neben dieser (scheinbaren) Einhegung der Blockaderegeln wurden zugleich jedoch mit einer königlichen Proklamation neue Konterbandelisten erlassen. Auf diesen wurden nun zahlreiche Artikel, die bisher zur bedingten Konterbande gezählt hatten, zu absoluter Konterbande aufgewertet. Zudem wurden neue Einträge erstellt. Deutlich spiegelte sich in diesen Veränderungen die bisherige Kriegserfahrung wieder, wenn beispielsweise Stacheldraht oder Entfernungsmessgeräte $\mathrm{zu}$ absoluter Konterbande erklärt wurden, ${ }^{66}$ war doch die Bedeutung der Artillerie früh im Ersten Weltkrieg erkennbar gewesen und wurde Stacheldraht nach dem Ende des Bewegungskriegs im Westen immer wichtiger.

Die britische Regierung versäumte es also nicht, die Liste der Konterbande den Gegebenheiten des Krieges anzupassen und ihr Dinge hinzuzufügen, die für die Kriegführung als wichtig erkannt worden waren. Dass das Foreign Office zugleich bemüht war, die Blockade so zu gestalten, dass gerade die Beziehungen

59 Beachte auch Lambert, Planning Armageddon (wie Anm. 5), S. 498.

60 Bell, A History of the Blockade (wie Anm. 11), S. 52 und S. 68f.; Osborne, Britain's Economic Blockade (wie Anm. 5), S. 65-67.

61 Hankey, The Supreme Command, vol. 1 (wie Anm. 16), S. 356f.; Cobb, Preparing for Blockade (wie Anm. 2), S. $252 \mathrm{f}$.

62 The Declaration of London Order in Council, No. 2, 1914, 29.10.1914, London Gazette, 27.10.1914, 2. Supplement, S. 8748 f.

63 Ebd.

64 Königliche Proklamation, 29.10.1914, London Gazette, 27.10.1914, 2. Supplement, S. $8747 \mathrm{f}$.

65 Chatterton, The Big Blockade (wie Anm. 17), S. $202 \mathrm{f}$.

66 Königliche Proklamation, 29.10.1914, London Gazette, 27.10.1914, 2. Supplement, S. 8747 f. 
zu den USA nicht zu sehr belastet würden, war dabei durchaus vorausschauende Außenpolitik: Großbritannien war auf den Handel mit neutralen Staaten angewiesen. Aus den USA bezog es beispielsweise einen wesentlichen Teil seiner Munition. ${ }^{67}$ Einen Kriegseintritt der USA auf deutscher Seite hätte es keinesfalls ertragen können - ein solcher war im Übrigen noch 1908 ein Szenario in den Kriegsplänen der Marine gewesen, galt den Zeitgenossen also nicht als ausgeschlossen. ${ }^{68}$ Doch auch, wenn man mit einiger Sicherheit bezweifeln kann, dass die USA Großbritannien aufgrund einer härteren Blockadepolitik den Krieg erklärt hätten, ${ }^{69}$ so lässt sich nicht verneinen, dass Großbritannien auf das Wohlwollen der USA angewiesen war; ${ }^{70}$ auch für die Durchsetzung der eigenen Blockade. Diese gewann mit dem Kriegseintritt der USA 1917 mehr als mit jedem anderen einzelnen Ereignis im Kriegsverlauf an Wirksamkeit und wurde zum ersten Mal »truly effective ${ }^{71}$ Schließlich waren zuvor die von den USA in neutrale Nachbarstaaten Deutschlands gelangenden Waren die größte verbliebene Schwachstelle der Blockade gewesen. ${ }^{72}$ Die Kooperationsbereitschaft der USA zu bewahren war also unbestreitbar eine solide außenpolitische Linie. ${ }^{73}$ Was auch den Zeitgenossen klar gewesen zu sein scheint, versicherten sie doch immer wieder, dass es Deutschlands größter Fehler gewesen sei, die USA durch die »unwavering stupidity of Imperial German statesmanship « zum Kriegseintritt gegen sich zu provozieren. ${ }^{74}$ Hankey formulierte, dass die schrittweise Loslösung von der Erklärung von London, die im Juli 1916 schließlich offiziell nicht mehr angewendet wurde, ${ }^{75}$ erst durch die gegnerischen Rechtsbrüche im Kriegsverlauf

67 Hankey, The Supreme Command, vol. 1 (wie Anm. 16), S. 358f.; Chatterton, The Big Blockade (wie Anm. 17), S. $237 \mathrm{f}$.

68 Grimes, Strategy (wie Anm. 5), S. 115.

69 Entsprechende Zweifel äußerte vor allem Jellicoe pointiert, Jellicoe an Fisher, 20.1.1916, zit. nach 1893-1916. Ed. by Temple A. Patterson, London 1966 (= The Jellicoe Papers. Selections from the private and official correspondence of Admiral of the Fleet Earl Jellicoe of Scapa, 1), S. 201.

70 Deutsche Diplomaten hofften zumindest bis 1916, dass ein Eingreifen der USA Großbritannien zum Frieden zwingen könnte, Osborne, Britain's Economic Blockade (wie Anm. 5), S. 156; beachte auch Lambert, Planning Armageddon (wie Anm. 5), S. 425 und S. 470-473.

71 Osborne, Britain's Economic Blockade (wie Anm. 5), S. 169; siehe auch ebd., S. 153; sowie Cobb, Preparing for Blockade (wie Anm. 2), S. 251f.; Halpern, World War I (wie Anm. 11), S. 101; Halpern, A Naval History (wie Anm. 9), S. 49 f.

72 Bell, A History of the Blockade (wie Anm. 11), S. 618, ferner ebd., 631-633; sowie Grove, Blockade (wie Anm. 4), S. 301.

73 Siehe auch Dirks, Julian S. Corbett (wie Anm. 2), S. 44.

74 David Lloyd George, War Memoirs, vol. 3, London 1934, S. 1672.

75 The Maritime Rights Order in Council, 1916, 7.7.1916, London Gazette, 7.7.1916, Supplement, S. 6821f. 
vertretbar wurde: "It was only the ruthless disregard of international law by the enemy which enabled us to jettison it on the plea of retaliation. ${ }^{76}$

Mit anderen Worten: Die im Kriegsverlauf als notwendig erkannten Verschärfungen und Ausweitungen der Blockade mussten so erfolgen, dass die USA ihre Unterstützung für Großbritannien deswegen nicht einstellten. Dementsprechend wurden wesentliche Veränderungen immer wieder so vorgenommen, dass sie als Reaktionen auf deutsche Affronts dargestellt werden konnten. ${ }^{77}$ Dies war durchaus kein Zufall. Fisher und der First Lord of the Admiralty Reginald McKenna hatten schon vor dem Krieg darauf gehofft, auf diese Weise eigene Abweichungen von den bestehenden Seerechtserklärungen kaschieren $\mathrm{zu}$ können, ${ }^{78}$ und in Deutschland war vor dem Krieg genau dies erwartet worden. ${ }^{79}$ Ein frühes Beispiel für dieses Vorgehen ist der Umgang mit gegnerischen Handelsschiffen in eigenen Häfen bei Kriegsbeginn. Diese wurden zunächst einmal beschlagnahmt. ${ }^{80}$ Die britische Regierung erklärte allerdings am 4. August 1914, ihnen eine zehntägige Schonfrist zu gewähren, in denen sie - ohne Konterbande - doch noch auslaufen dürften, wenn Deutschland eine vergleichbare Versicherung bis zum 7. August abgebe. ${ }^{81}$ An diesem Tag verkündete dann Außenminister Edward Grey, eine entsprechende Versicherung nicht erhalten zu haben, weshalb man selbst auf die Schonfrist verzichten werde. ${ }^{82}$ Im Kriegsverlauf wurde dieses Handlungsmuster regelmäßig genutzt. Als Großbritanniens Führung weite Teile der Nordsee am 2. November 1914 zum Kriegsgebiet erklärte und neutralen Schiffen Routen nahe der eigenen Küste zuwies, während alle anderen Bereiche nicht sicher seien, begründete sie diesen Schritt damit, dass Deutschland diese Seegebiete durch Seeminen längst »in a more deadly fashion« geschlossen hätte. ${ }^{83}$ Der nächste wichtige britische Schritt in der Eskalationsspirale erfolgte im März 1915. Die

76 Hankey, The Supreme Command, vol. 2 (wie Anm. 16), S. 548; siehe auch Osborne, Britain's Economic Blockade (wie Anm. 5), S. $131 \mathrm{f}$.

77 Die deutsche Regierung begründete ihr Vorgehen freilich ebenfalls mit britischen Rechtsbrüchen, beachte beispielsweise Carl Hollweg, Unser Recht auf den U-Bootskrieg, Berlin 1917, S. 17 f.; ferner Bühler, Neutralität (wie Anm. 45), S. 39.

78 Beachte hierzu Tagebucheinträge Grant Duff, 22. und 24.2.1911, zit. bei Offer, The First World War (wie Anm. 10), S. 280.

79 Die militärische und rechtliche Bedeutung der Blockade. In: 1914 (wie Anm. 20), S. 279-308, hier S. 301f. und S. 308.

80 Für Deutschland siehe Tagebucheintrag Almeric Fitzroy, 1.8.1914, zit. nach Sir Almeric Fitzroy, Memoirs, vol. 2, 5. ed., London 1925, S. 558f.; für Großbritannien siehe Eintrag vom 6.8., ebd., S. 560 .

81 Königliche Anordnung, 4.8.1914, London Gazette, 5.8.1914, Supplement, S. 6162f.

82 Notiz, 7.8.1914, London Gazette, 8.8.1914, Supplement, S. 6281f.

83 Hawkins, The Starvation Blockades (wie Anm. 33), S. 63-65, Zitat S. 63. 
Londoner Regierung kündigte an, keinem Handelsschiff mehr zu erlauben, deutsche Häfen anzulaufen. Schiffe würden einen Pass erhalten, um neutrale oder alliierte Häfen anzulaufen. Waren, die nicht durch solche Papiere gedeckt sein, müssten in britischen Häfen entladen werden. Auch werde man kein Handelsschiff mit Waren passieren lassen, die es in einem deutschen Hafen geladen habe. Schließlich würde man auch Schiffe beschlagnahmen, die ihre Pässe missbrauchten, um dennoch Handel mit Deutschland zu betreiben. Wer allerdings versicherte, keine Waren mehr für Deutschland zu transportieren, dessen Schiffe könnten von dem System ausgenommen werden. ${ }^{84}$ Gerechtfertigt wurden diese drastischen Maßnahmen damit, dass Deutschland die Gewässer um Großbritannien - als Reaktion auf die britische Erklärung vom 2. November ${ }^{85}$ - zum Kriegsgebiet für seine U-Boote erklärt hatte. Tatsächlich hatte die deutsche Erklärung den Zorn gerade der USA von der britischen Blockade abgelenkt. ${ }^{86}$ Die britische Regierung argumentierte nun, der deutsche Schritt sei eine »violation of the usages of war « und gebe ihr »an unquestionable right of retaliation«. Man werde die eigenen verschärften Maßnahmen jedoch »without risk to neutral ships or to neutral or non-combatant life, and in strict observance of the dictates of humanity « ergreifen. ${ }^{87}$ Hankey bestätigte in seinen Erinnerungen, dass man den deutschen Schritt als "pretext to strike off yet another of the fetters which were hampering the full exercise of our sea-power « genutzt habe. ${ }^{88}$ In der Forschung gilt diese »Reprisals Order-in-Council« im Allgemeinen als entscheidender Schritt hin zu einer wirksamen Blockade. ${ }^{89}$ Fest steht, dass im März 1915 zahlreiche Schiffe begannen, von sich aus die britischen Häfen zu Kontrollen anzulaufen und weniger versuchten, die britischen Patrouillen zu umgehen. ${ }^{90}$

Anders als derartige Änderungen an der Schärfe der Blockade wurden Erweiterungen der Konterbandeliste in aller Regel nicht gesondert begründet. Sie wurden allerdings regelmäßig infolge der Kriegserfahrungen getätigt, wenn es als »expedient « erkannt worden war, die bestehenden Listen zu erweitern. ${ }^{91}$ So wurden am 27. Mai 1915, kurz nach der Munitionskrise, »Lathes and other

84 Reprisals Order in Council, 11.3.1915, London Gazette, 15.3.1915, 2. Supplement, S. 2605 f.

85 Bühler, Neutralität (wie Anm. 45), S. 39.

86 Chatterton, The Big Blockade (wie Anm. 17), S. 96f.

87 Reprisals Order in Council, 11.3.1915, London Gazette, 15.3.1915, 2. Supplement, S. 2605 f.

88 Hankey, The Supreme Command, vol. 1 (wie Anm. 16), S. 368; ebenso vol. 2, S. 488 f.

89 Chatterton, The Big Blockade (wie Anm. 17), S. 99-101; Osborne, Britain's Economic Blockade (wie Anm. 5), S. 88.

90 Chatterton, The Big Blockade (wie Anm. 17), S. 108f. und S. $139 \mathrm{f}$.

91 So die übliche Formulierung, siehe z.B. Proklamation einer Veränderung der Konterbandeliste, 23.12.1914, London Gazette, 23.12.1914, Supplement, S. $11017 \mathrm{f}$. Beachte auch Tagebucheintrag Fitzroy, 19.9.1914, zit. nach Fitzroy, Memoirs (wie Anm. 80), S. 571. 
machines or machine-tools capable of being employed in the manufacture of munitions of war « der Liste der absoluten Konterbande hinzugefügt. ${ }^{92}$ Etwas komplizierter stellte sich die Lage dar, als Baumwolle am 20. August 1915 in die Liste aufgenommen wurde. Zwar hieß es auch hier in der Proklamation nur, dass der Schritt als »expedient" erkannt worden sei. ${ }^{93}$ Tatsächlich aber hatte er eine längere Vorgeschichte. Baumwolle war ein wichtiger Rohstoff für die Munitionsproduktion. ${ }^{94}$ Nicht zuletzt deshalb forderte die britische Presse im Sommer 1915 zunehmend lautstark, sie zu Konterbande zu erklären. ${ }^{95}$ Da allerdings Baumwolle für die Südstaaten der USA ein wichtiges Exportgut war, wollte es die britische Regierung nicht riskieren, die Konterbandeliste unilateral und unangekündigt entsprechend auszuweiten. Stattdessen bemühte sie sich über den Sommer hinweg in Verhandlungen mit den USA, diesen Schritt diplomatisch vorzubereiten und zu erklären. Erst nachdem das gelungen war, wurde Baumwolle zur Konterbande erklärt. ${ }^{96}$ Dass die Erklärung erfolgte, einen Tag nachdem deutsche U-Boote die >Arabic versenkt hatten, was erneut zu diplomatischen Spannungen zwischen Deutschland und den USA führte, war ein bloßer Zufall. ${ }^{97}$ Daraus allerdings wie Chatterton abzuleiten, dass Deutschlands Auftreten gegenüber den USA zeige, dass auch Großbritannien hätte unverfrorener auftreten können, ohne schwerwiegende Komplikationen erwarten zu müssen, ist blauäugig angesichts der Bedeutung, die zugleich der ungeschickten deutschen Diplomatie beim Kriegseintritt der USA zugeschrieben wurde - ein Ergebnis, das das schrittweise Vorgehen des Foreign Office mehr als rechtfertigte. ${ }^{98}$

Im weiteren Kriegsverlauf wurden die Konterbandelisten noch mehrfach erweitert, wobei allein schon die Versuche, dem Gegner Munition und Sprengstoffe vorzuenthalten, einen veritablen Rattenschwanz an gesperrten Artikeln nach sich zogen. So fand sich unter zahlreichen entsprechend begründeten Einträgen in der Liste vom 14. Oktober 1915 beispielsweise folgender:

»Materials used in the manufacture of explosives, including: - Nitric acid and nitrates of all kinds; sulphuric acids; fuming sulphuric acid (oleum); acetic acid and acetates; barium chlorate and perchlorate; calcium acetate, nitrate and carbide; potassium salts and caustic potash; ammonium salts and ammonia liquor; caustic soda, sodium chlorate and perchlo-

92 Proklamation über geänderte Konterbandelisten, 27.5.1915, London Gazette, 28.5.1915, S. 5100.

93 Proklamation über geänderte Konterbandelisten, 20.8.1915, London Gazette, 20.8.1915, S. 29271.

94 Bell, A History of the Blockade (wie Anm. 11), S. 309f.

95 Chatterton, The Big Blockade (wie Anm. 17), S. 147 und S. $187 \mathrm{f}$.

96 Bell, A History of the Blockade (wie Anm. 11), S. 313-316.

97 1893-1916 (wie Anm. 69), S. 138.

98 Chatterton, The Big Blockade (wie Anm. 17), S. 188. 
rate; mercury; benzol, toluol, xylol, solvent naphtha, phenol (carbolic acid), cresol, naphthalene, and their mixtures and derivatives; aniline, and its derivatives; glycerine; acetone; acetic ether; ethyl alcohol; methyl alcohol; ether; sulphur; urea; cyanamide; celluloid.«99

Über neue Listen am 27. Januar, 12. April, 27. Juni, 3. Oktober, 23. November und 29. Dezember 1916 erreichten die britischen Konterbandelisten schließlich den für die restliche Kriegszeit gültigen, »consolidate[d]« Stand vom 2. Juli 1917, wobei die Erweiterungen immer wieder als »expedient« erklärt wurden und die Erfahrungen mit dem fortdauernden und sich totalisierenden Krieg einerseits, die diplomatischen Möglichkeiten andererseits widerspiegelten. ${ }^{100}$ In ihrer letzten Form umfassten die Listen insgesamt 217 Einträge. Einige davon waren recht begrenzt, zum Beispiel »Peppers« oder führten ähnliche Varianten auf, wie »Haematite iron ore« und »Haematite pig iron«. Andere dagegen waren umfangreicher, zum Beispiel:

»Gold, silver, paper-money, securities, negotiable instruments, cheques, drafts, orders, warrants, coupons, letters of credit, delegation, or advice, credit and debit notes, or other documents which in themselves, or if completed, or if acted upon by the recipient authorise, confirm, or give effect to the transfer of money, credit, or securities. ${ }^{101}$

Insgesamt wurde ein äußerst breites Spektrum an Waren abgedeckt, bis hin zu »Bones in any form, whole or crushed; bone ash ${ }^{102}$ In jedem Fall war es ein Umfang, der ohne die Erfahrungen des Krieges und ohne die schrittweise diplomatische Entwicklung kaum denkbar gewesen wäre.

Auch die weiteren Verschärfungen der Blockade waren von den gesammelten Kriegserfahrungen abhängig - und hätten nicht bei Kriegsbeginn aus dem Nichts erschaffen werden können. Besonders deutlich wird dies an »The Declaration of London Order in Council, 1916«, mit der am 30. März 1916 erklärt wurde, dass man bedingte Konterbande auch dann als für den Gegner bestimmt ansehen werde, wenn der Empfänger im Kriegsverlauf bereits einmal Konterbande an den Gegner geliefert habe. ${ }^{103}$ Um eine solche Regel durchzusetzen, mussten detail-

99 Proklamation über geänderte Konterbandelisten, 14.10.1915, London Gazette, 15.10.1915, S. $10131 \mathrm{f}$.

100 Proklamation über geänderte Konterbandelisten, 2.7.1917, London Gazette, 3.7.1917, S. 6541f. 101 Königliche Proklamation der Konterbande, 2.7.1917, London Gazette, 3.7.1917, S. 6541-6544. Die Liste blieb bis zum Waffenstillstand gültig, siehe Bell, A History of the Blockade (wie Anm. 11), S. 740 .

102 Ebd.

103 The Declaration of London Order in Council, 1916, 30.3.1916, London Gazette, 30.3.1916, Supplement, S. 3404. 
lierte Informationen über die weltweiten Handelsnetze vorliegen, die erst infolge des Krieges in diesem Maße erstellt wurden. ${ }^{104}$ Vor Kriegsbeginn hatte sich zunächst zwar bereits das 1887 gegründete Naval Intelligence Department (NID) mit solchen Fragen befasst, das routinemäßig Analysen darüber erstellte, wie etwaigen Gegnern mit Blockaden geschadet werden könnte. ${ }^{105}$ Diese blieben jedoch eher allgemeiner Art. So meinte der Director of Naval Intelligence Charles Ottley 1905, da Deutschlands Außenhandel zu 60 Prozent über See verlaufe, "within six weeks of the declaration of war, at least one-half of Germany's trade would have been snatched from her. ${ }^{106}$ Die Details blieben allerdings vage. Hankey, Mitglied im Ballard-Komitee, das Fisher Ende 1906 einsetzte, um Kriegspläne gegen Deutschland zu erarbeiten, ${ }^{107}$ erinnerte sich später: »We [...] could not judge whether it would be possible to squeeze her into submission, or how long it would take, particularly in view of the assistance she could obtain from her continental neighbours. ${ }^{108}$ Es fehlte vor dem Krieg an den notwendigen Erfahrungen, um die erhobenen Daten einordnen zu können. Dennoch entstand ein rudimentäres Netzwerk, auf dessen Basis die Informationssammlung im Krieg fußen konnte. Namentlich regte der im August 1906 zum Chef der Trade Division des NID ernannte Kapitän zur See Henry Hervey Campbell an, Handelsschiffe systematisch Informationen sammeln $\mathrm{zu}$ lassen, die durch Zollbeamte ausgewertet werden sollten. Obwohl sich Fisher gegen den Plan stellte, nahm ihn McKenna im Mai 1909 an. Allerdings wurde mit der Umsetzung auch aus finanziellen Gründen erst 1911 begonnen. In diesem Jahr wurden drei neue Posten im britischen Konsulardienst geschaffen: In St. Vincent, auf den Kapverdischen Inseln und in Montevideo wurden ehemalige Marineoffiziere stationiert, die deutsche Schiffe beobachten sollten, die im Kriegsfall bewaffnet und zu Angriffen auf den britischen Handel verwendet werden könnten. ${ }^{109}$ Als der Krieg im August 1914 begann, wusste die Admiralty angeblich um den Aufenthaltsort fast jedes deutschen Schiffes über 300 t. $^{110}$

Dieses Wissen war für die Blockade während des Kriegsverlaufs jedoch von sekundärer Bedeutung, da die im Ausland befindlichen deutschen Schiffe mit

104 Beachte auch Lambert, Planning Armageddon (wie Anm. 5), S. 500.

105 Zum NID, mit Fokus auf der Blockade, siehe vor allem Grimes, Strategy (wie Anm. 5); sowie Cobb, Preparing for Blockade (wie Anm. 2).

106 Ottley an Corbett, 1.7.1905, zit. nach Grimes, Strategy (wie Anm. 5), S. 64f.; siehe auch Lambert, Planning Armageddon (wie Anm. 5), S. 40.

107 Grimes, Strategy (wie Anm. 5), S. 84-86.

108 Hankey, The Supreme Command, vol. 1 (wie Anm. 16), S. 40.

109 Seligmann, The Royal Navy (wie Anm. 5), S. 103f., 109, 114f. und S. 117-120.

110 Tagebucheintrag Fitzroy, 9.8.1914, zit. nach Fitzroy, Memoirs (wie Anm. 80), S. 564 f. 
Kriegsbeginn aus Furcht vor einer Beschlagnahme durch die britische Marine, die die Seehandelswege kontrollierte, im Wesentlichen in den Häfen blieben. ${ }^{111} \mathrm{Im}$ Krieg wurde die Informationssammlung daher über die deutschen Schiffe hinaus ausgedehnt, auf neutrale Schiffe zum einen und auf Firmen zum anderen. ${ }^{112}$ So wurden mithilfe der Postzensur Schiffe enttarnt, die als Blockadebrecher dienten; einmal erkannt, wurden diese in schwarzen Listen erfasst und konnten fortan gezielt abgefangen und zur Kontrolle in britische Häfen geschickt oder mit härteren Strafmaßnahmen belegt werden. ${ }^{113}$

Die entsprechenden Listen sind ein hervorragendes Beispiel für eine Tätigkeit, die den Krieg und die in ihm gesammelten Erfahrungen erforderte und die schlicht nicht vorbereitet werden konnte. Ähnliches gilt für eine Liste deutscher Scheinfirmen, die das Foreign Office ab Oktober 1914 erstellte, um entsprechende Waren zu blockieren. ${ }^{114}$ Aber auch über legitime neutrale Firmen legte das Foreign Office Karteien an. Hier wurden nicht zuletzt die üblichen Warenarten und mengen verzeichnet, um anhand größerer Veränderungen zu erkennen, ob die Firmen zusätzliche Waren für den Handel mit Deutschland erwarben. ${ }^{115}$ Anfang 1915 entstand für diese Zwecke im Foreign Office eine spezielle Statistiksektion, die im April 1916 zum War Trade Statistical Department aufgewertet wurde. ${ }^{16}$ Auch die über Deutschland selbst gesammelten Informationen wurden im Krieg präzisiert. So wusste das Foreign Office Anfang März 1915 beispielsweise, welche Stoffe in Deutschland inzwischen seltener oder teurer geworden waren, und stellte Schätzungen darüber an, welchen Anteil an den Veränderungen die Blockade hatte. Im Fall von Nahrungsmitteln wurde ihr eine achtprozentige Preissteigerung zugeschrieben - während schlechten Ernteergebnissen 20 Prozent angelastet wurden. ${ }^{117}$ Auf Basis solcher Erkenntnisse beriet die Trade Division das Contraband Committee hinsichtlich seiner Entscheidungen darüber, ob einzelne Schiffe freigelassen oder vor ein Prisengericht gebracht werden sollten. ${ }^{118}$

111 Hankey, The Supreme Command, vol. 1 (wie Anm. 16), S. 180.

112 Siehe beispielsweise zur Informationssammlung über Handelsschiffe, die aus den USA nach Nordeuropa fuhren, Lambert, Planning Armageddon (wie Anm. 5), S. 376.

113 Chatterton, The Big Blockade (wie Anm. 17), S. 172 und S. 208.

114 Osborne, Britain's Economic Blockade (wie Anm. 5), S. 68-72; beachte auch Lambert, Planning Armageddon (wie Anm. 5), S. 14.

115 Chatterton, The Big Blockade (wie Anm. 17), S. $108 \mathrm{f}$.

116 British Foreign Policy under Sir Edward Grey. Ed. by Francis Harry Hinsley, Cambridge 1977, S. 653 , Anm. 69.

117 Tagebucheintrag Fitzroy, 2.3.1915, zit. nach Fitzroy, Memoirs (wie Anm. 80), S. 585 f.

118 Chair, The Sea is Strong (wie Anm. 13), S. $207 \mathrm{f}$. 
Die inhaltliche Gestaltung der Blockade, sowohl was die Zusammensetzung der Konterbandelisten als auch den jeweiligen Umgang mit den verschiedenen Kategorien von Waren, das Verhalten gegenüber unkooperativen Schiffseignern und andere Fragen der Schärfe der Blockade angeht, wurde also im Ersten Weltkrieg auf Basis der Kriegserfahrungen und vor dem Hintergrund der diplomatischen Möglichkeiten über den Stand hinaus erweitert, der zu Kriegsbeginn denk- und legitimierbar war; ja sie konnte erst aufgrund der Erfahrungen des Krieges und der Entwicklung der diplomatischen Lage im Kriegsverlauf entsprechend erweitert werden. Im Folgenden soll gezeigt werden, dass der Erste Weltkrieg auch auf die Methodik der Blockade als Katalysator wirkte. Der Abschnitt beginnt mit den Schiffen, welche die Blockade im Norden des britischen »Wellenbrechers« umsetzen sollten; die routiniert den gesamten Krieg über stattfindende Kontrolle des Ärmelkanals und Mittelmeers bleiben an dieser Stelle außen vor.

\section{Von alten Kreuzern zu Schreibtischen - Neue Methoden zur Durchsetzung der Blockade}

Durchgesetzt wurden die im Kriegsverlauf auch für die neutralen Staaten in Deutschlands Nachbarschaft zunehmend scharfen Bedingungen der Blockade zunächst mithilfe von Kriegsschiffen; letztlich also so, wie Blockaden in der (Rechts-)Praxis des 19. Jahrhunderts entworfen worden waren. Dass sich diese Form im Kriegsverlauf dramatisch verändern würde, dass Schiffe letztlich beinahe überflüssig werden würden, war zunächst nicht abzusehen. Ganz im Gegenteil: Schon am 1. August 1914 wurden acht zwischen 1890 und 1892 gebaute Kreuzer der >Edgar<-Klasse reaktiviert. Sie hatten der Royal Navy in Friedenszeiten als Trainingsgeschwader gedient und sollten nun als 10th Cruiser Squadron die Seehandelswege im Norden des »britischen Wellenbrechers» kontrollieren. ${ }^{119}$

Am 3. August verließ der Chef des 10. Kreuzergeschwaders, Konteradmiral Dudley R.S. de Chair, mit seinem Flaggschiff `Crescent` den Hafen von Portsmouth. Die übrigen Schiffe seines Geschwaders folgten später, da es aufgrund des Bank Holiday am 3. August zu Verzögerungen im Bahnverkehr gekommen war und nicht alle Reservisten und aus dem Ruhestand zurückgeholten Offiziere

119 Hawkins, The Starvation Blockades (wie Anm. 33), S. 16; Chatterton, The Big Blockade (wie Anm. 17), S. 32-34. 
rechtzeitig vor Ort waren. ${ }^{120}$ Ganz so, wie es den hergebrachten Vorstellungen von Blockaden entsprach, sollte es die Aufgabe der Kriegsschiffe sein, deutsche Kriegs- und Handelsschiffe abzufangen, »and sink or capture them; also to stop all neutral vessels proceeding to German ports, and to deny the anchorage of any harbour in the Shetlands or Orkneys to the enemy «. ${ }^{121}$ Bereits am 5. August konnte de Chairs Geschwader den ersten Erfolg feiern, als es die deutsche >Wilhelm Behrens aufbrachte und in den Hafen von Clyde schickte. ${ }^{122}$ Drei Tage später war das Blockadegeschwader vollständig auf Station. ${ }^{123}$ Für den Admiral begannen der Krieg und die ihm anvertraute Blockade erfolgreich: "The mobilization of the Fleet was really a great success [...] So far as requirements had been foreseen, the Admiralty had done well. «124 Auch Hankey fasste später zufrieden zusammen, mit Kriegsbeginn »the German mercantile flag at once disappeared from the high seas. ${ }^{125}$

Allerdings gab es deutlichen Spielraum für Verbesserungen, was zunächst hieß, die Anzahl der Schiffe im Blockadegebiet zu erhöhen und dieses intensiver zu kontrollieren. So unterband die Admiralty zum einen Mitte August die Praxis, Kreuzer für Einsätze der Grand Fleet aus der Blockadelinie abzuziehen. Damit entschied sie, entgegen der Überlegung Fishers vor dem Krieg, dass die wirtschaftliche Blockade Deutschlands nicht den Versuchen untergeordnet werden durfte, seine Hochseeflotte zum Kampf zu stellen; eine Entscheidung, die der Befehlshaber der Grand Fleet, Admiral Sir John Jellicoe, laut de Chair teilte. ${ }^{126}$ Anfang Oktober kamen Jellicoe und Churchill gar überein, dass die Grand Fleet im Bedarfsfall das Blockadegeschwader unterstützen sollte. ${ }^{127}$ Sie rückten also die Blockade dezidiert in den Mittelpunkt der britischen Seestrategie, stellten sie letztlich über die Konfrontation der Flotten. ${ }^{128}$

120 Chair, The Sea is Strong (wie Anm. 13), S. 160 f.; Cobb, Preparing for Blockade (wie Anm. 2), S. 241f.; Chatterton, The Big Blockade (wie Anm. 17), S. 32-35.

121 Chair, The Sea is Strong (wie Anm. 13), S. 167.

122 Rear-Admiral de Chair an Adiralty, HMS Crescent, 27.8.1914, Report of Proceedings, 1.-27.8.1914, abgedr. in The Maritime Blockade (wie Anm. 36), S. 26-32, hier S. 26f.; Chair, The Sea is Strong (wie Anm. 13), S. 163; Cobb, Preparing for Blockade (wie Anm. 2), S. XVIII; Chatterton, The Big Blockade (wie Anm. 17), S. 33-35.

123 Cobb, Preparing for Blockade (wie Anm. 2), S. $241 \mathrm{f}$.

124 Chair, The Sea is Strong (wie Anm. 13), S. 161; er war allerdings der Ansicht, dass es besser gewesen wäre, noch früher eingesetzt zu werden, siehe ebd., S. 169.

125 Hankey, The Supreme Command, vol. 1 (wie Anm. 16), S. 180.

126 Chair, The Sea is Strong (wie Anm. 13), S. 171 und S. $176 \mathrm{f}$.

127 Churchill an Jellicoe, 8.10.1914, zit. nach 1893-1916 (wie Anm. 69), S. 73f.; Winston S. Churchill, 1911-1914, Leipzig 1924 (= Weltkrisis, 1), S. 305.

128 Siehe auch Dirks, Julian S. Corbett (wie Anm. 2), S. 42. 
Zum anderen wurde das 10. Kreuzergeschwader verstärkt. Schon vor Kriegsbeginn hatte der Plan bestanden, die Blockadelinien durch bewaffnete Handelsschiffe zu ergänzen, um die langen Wege zu den Nachschubbasen und die notwendigen Wartungszeiten der im Dauereinsatz befindlichen Schiffe auszugleichen. Entsprechende Vorhaben waren in den Jahren vor dem Krieg Teil der Kriegspläne der Admiralty gewesen. ${ }^{129}$ Konkret hatte die Admiralty im April 1914 geplant, innerhalb der ersten drei Wochen eines Krieges fünf Hilfskreuzer im Norden einzusetzen; für mehr könnten innerhalb dieses Zeitfensters keine Besatzungen gefunden werden. ${ }^{130}$ Angesichts der internationalen Spannungen hatte Churchill bereits am 28. Juli angeordnet, das neueste Passagierschiff der CunardLine, die >Aquitania`, die wie ihre Schwesterschiffe `Lusitania` und `Mauretania schon bei der Konstruktion auf entsprechende Umbauten vorbereitet worden war, als Hilfskreuzer auszurüsten. ${ }^{131}$ Am 3. August, dem Tag, an dem de Chair aus Portsmouth auslief, requirierte die britische Regierung zudem erste Handelsschiffe. ${ }^{132}$ Noch im selben Monat stießen erstmals vier dieser Schiffe zum Blockadegeschwader, beginnend mit der >Alsatian` am 18. August. ${ }^{133}$

Wenige Wochen später wurde eine Veränderung des Blockadegeschwaders notwendig, die so nicht vorhergesehen worden war. Die alten Kreuzer nämlich erwiesen sich den Bedingungen der nördlichen Nordsee, noch dazu in Herbst und Winter, als nicht gewachsen. In einem Sturm am 11. November wurden die Schiffe schwer beschädigt. Die notwendigen Reparaturen waren so umfangreich, dass die Admiralty beschloss, die betagten Kreuzer durch besser geeignete Schiffe zu ersetzen. Die »Northern Patrol« sollte fortan aus 24 »armed liners« bestehen. Bereits am 17. November seien alle erforderlichen Schiffe seitens der Admiralty eingezogen gewesen und schon drei Tage später wurden die alten Kreuzer außer Dienst gestellt. Am 2. Dezember betrat de Chair sein neues Flaggschiff, die >Alsatian<, die zwischenzeitlich mit schweren Waffen ausgerüstet worden war. ${ }^{134}$ Noch

129 Cobb, Preparing for Blockade (wie Anm. 2), S. 97 f.; Schreiben Admiral Sir George Callaghans vom 2.10.1913, zit. nach 1902-1918 (wie Anm. 15), S. 82-90, hier S. 85 f.

130 Cobb, Preparing for Blockade (wie Anm. 2), S. XIXf.

131 Die modernen Passagierschiffe wurden jedoch bald re-zivilisiert, da sie zu viel Treibstoff verbrauchten, um sie als Hilfskreuzer einzusetzen, Seligmann, The Royal Navy (wie Anm. 5), S. $165 f$.

132 Hankey, The Supreme Command, vol. 1 (wie Anm. 16), S. 159. Cobb nennt den 11. August, Cobb, Preparing for Blockade (wie Anm. 2), S. $244 \mathrm{f}$.

133 Chatterton, The Big Blockade (wie Anm. 17), S. 60f.; 1893-1916 (wie Anm. 69), S. 51 und S. 245. Chair verweist nur auf drei Schiffe in diesem Monat, Chair, The Sea is Strong (wie Anm. 13), S. 173.

134 Chair, The Sea is Strong (wie Anm. 13), S. 180-182; Chatterton, The Big Blockade (wie Anm. 17), S. 52-55 und S. 65. 
im selben Monat war die Mehrzahl der vorgesehenen Schiffe im Einsatz, ${ }^{135}$ spätestens im Juli 1915 alle $24 .{ }^{136}$

Die britische Blockade hatte damit die Form angenommen, die für die meiste Zeit des Ersten Weltkriegs kennzeichnend war. Die aus umgerüsteten zivilen Schiffen bestehende »Northern Patrol« fing in den Seegebieten zwischen Großbritannien, Norwegen und Island Schiffe ab, die versuchten, unerwünschte Waren oder Personen nach Deutschland zu transportieren, während die Grand Fleet sie vor größeren deutschen Angriffen abschirmte. ${ }^{137}$ Über zwei Jahre hinweg veränderte sich dieses Arrangement praktisch kaum, sieht man davon ab, dass die französische Marine Ende 1915 auf britischen Wunsch hin begann, sich an der Patrouille zu beteiligen. Das Ziel dieser Beteiligung war es, den außenpolitischen Druck auf Großbritannien abzufedern; die Schiffe, die hierfür verwendet wurden, stellte bald Großbritannien, da sich die verfügbaren französischen Einheiten für den Einsatz im Norden schnell als ungeeignet erwiesen hatten. ${ }^{138}$ Davon abgesehen war die bemerkenswerteste Veränderung ein Kommandowechsel: Ende Februar 1916 wurde die >Alsatian` nach Liverpool beordert und de Chair als Marineberater Lord Robert Cecils ins Contraband Department des Foreign Office versetzt. Sein Nachfolger wurde Vizeadmiral Reginald Tupper, der seine Flagge am 6. März auf der >Alsatian`setzte. ${ }^{139}$

Die einschneidendste Veränderung der inzwischen von Großbritannien, Frankreich und den USA getragenen Blockade Deutschlands erfolgte im Herbst und Winter 1917/18: Das 10. Kreuzergeschwader wurde aufgelöst. ${ }^{140}$ Die Schiffe, die bisher dazu gedient hatten, die Wirtschaft Deutschlands anzugreifen, wurden nun eingesetzt, um die britische Wirtschaft zu schützen. Namentlich dienten sie

135 Chair zählte am 29.12.1914 16 Schiffe auf Patrouille, Chair, The Sea is Strong (wie Anm. 13), S. 188; Chatterton, The Big Blockade (wie Anm.17), S. 58f., nennt 19; Osborne, Britain's Economic Blockade (wie Anm. 5), S. 72f., spricht von 23; Cobb, Preparing for Blockade (wie Anm. 2), S. 311-313, führt für den Februar 191521 bewaffnete Handelsschiffe auf.

136 Chatterton, The Big Blockade (wie Anm. 17), S. 171.

137 Vergleiche Cobb, Preparing for Blockade (wie Anm. 2), S. $258 \mathrm{f}$.

138 Chatterton, The Big Blockade (wie Anm. 17), S. 210; Osborne, Britain's Economic Blockade (wie Anm. 5), S. 104.

139 Chatterton, The Big Blockade (wie Anm. 17), S. $245 \mathrm{f}$.

140 Grove spricht von Ende Januar 1918, Grove, Blockade (wie Anm. 4), S. 301; Halpern nennt bereits den November 1917, Halpern, A Naval History (wie Anm. 9), S. 49f.; Osborne kennt den 8.12.1917 als Datum der Auflösung, Osborne, Britain's Economic Blockade (wie Anm. 5), S. 166; Admiral Tupper spricht von einer schrittweisen Auflösung ab Mitte 1917, die im Januar 1918 abgeschlossen war, als er einen anderen Posten antrat, Tupper, Reminiscences (wie Anm. 14), S. 264. Diese Beschreibung wird durch zahlreiche Quellen gestützt, die sich bei The Maritime Blockade (wie Anm. 36) finden, beispielsweise die Dokumente 361, 366, 397, 399, 404, 405, 406 und 408. 
als Begleitschutz für Konvois nach Großbritannien, um diese vor den Angriffen deutscher U-Boote zu bewahren. ${ }^{141}$ Dass die alliierte Blockade dennoch nichts von ihrer Wirkung verlor, lag daran, dass es der britischen Außenpolitik im Kriegsverlauf gelungen war, neben den maritimen Machtmitteln weitere Druckmittel zu ihrer Durchsetzung zu etablieren. ${ }^{142}$

Dass die Blockade nicht allein auf der Arbeit der Blockadegeschwader beruhen würde, deutete sich schon Anfang August 1914 an, als die Niederlande den Nahrungsmittelhandel mit Deutschland verboten - ein Verbot, das freilich zunächst kaum durchgesetzt wurde. ${ }^{143}$ Der britischen Regierung allerdings lag viel daran, den Weiterverkauf von Nahrungsmitteln aus neutralen Staaten in Nordeuropa an Deutschland zu verhindern. Dies galt umso mehr, nachdem sie Ende Oktober die Regeln der continuous voyage für bedingte Konterbande wieder hatte einschränken müssen. Als ein Weg, dieses Ziel zu erreichen, boten sich Vereinbarungen mit den neutralen Staaten, beziehungsweise mit den dortigen Händlern an. Schon am 14. August hatte das Restriction of Enemy Supplies Committee beschlossen, den Niederlanden zu versprechen, die Kohlezufuhr des Landes zu garantieren, wenn es im Gegenzug keine Nahrung nach Deutschland liefere. ${ }^{144}$ Formalisiert wurde dieses Konzept einer freiwilligen Beschränkung der jeweiligen Importe auf das für die eigene Versorgung notwendige Maß in Übereinkünften mit privaten Verbünden wie dem Netherlands Overseas Trust im November 1914 und weiteren, ähnlichen Organisationen, die während des Krieges in anderen neutralen Staaten entstanden, ${ }^{145}$ beispielsweise der Sociètè de Surveillance Suisse. ${ }^{146}$ Den entsprechenden Vereinigungen wurde der Handel mit Konterbande erlaubt, wenn sie garantierten, diese nicht nach Deutschland zu exportieren. Um seine Ansprüche durchzusetzen, griff Großbritannien auf sein weltweites Netz an Marinestützpunkten und die eigenen Kohlevorkommen zurück: Nur kooperationsbereite Firmen erhielten hierauf Zugriff. ${ }^{147}$ Damit wurde ein Plan umgesetzt, den

141 Chatterton, The Big Blockade (wie Anm. 17), S. 279.

142 Beachte Memorandum für den Commander-in-Chief, 6.11.1917, abgedr. in The Maritime Blockade (wie Anm. 36), S. 771-774, besonders S. 773.

143 Russell, Prize Courts (wie Anm. 5), S. $80 \mathrm{f}$.

144 David French, British Economic and Strategic Planning, 1905-1915, Milton Park 2006, Reprint von 1982, S. 114.

145 Hankey, The Supreme Command, vol. 1 (wie Anm. 16), S. 357; Osborne, Britain's Economic Blockade (wie Anm. 5), S. 76f.; beachte ferner Lambert, Planning Armageddon (wie Anm. 5), S. 468 .

146 Bell, A History of the Blockade (wie Anm. 11), S. 299-308, 454 und S. 503-521; Osborne, Britain's Economic Blockade (wie Anm. 5), S. 93-96.

147 Hankey, The Supreme Command, vol. 1 (wie Anm. 16), S. 358. 
das Committee of Imperial Defence bereits 1912 gefasst hatte. ${ }^{148}$ Tatsächlich erwies sich die britische Kohle im Kriegsverlauf als äußerst wirkungsvolles Druckmittel, um die gewünschte Rationierung der Importe neutraler Staaten zu erzwingen. Allerdings war diese Wirksamkeit nicht von Kriegsbeginn an in vollem Umfang gegeben, da zunächst auch Deutschland Kohle an die neutralen Länder in seiner Nachbarschaft liefern konnte und zudem eine wichtige Quelle für bestimmte Industrieprodukte wie beispielsweise Färbemittel war. ${ }^{149}$ Nach und nach jedoch setzte sich Großbritannien mit seinen Alliierten durch, denn je größer deren Anzahl wurde, desto wichtiger waren sie in Summe als Handelspartner für die verbleibenden neutralen Nachbarländer Deutschlands. Diesen Umstand nutzte die britische Regierung nicht zuletzt dadurch, dass sie weitere wichtige Waren neben der Kohle identifizierte und den entsprechenden eigenen Handel mit den neutralen Ländern von Konzessionen abhängig machte. ${ }^{150}$ Hankey beschreibt dieses Vorgehen, das unter der Leitung des im Februar 1916 gegründeten Ministry of Blockade zentralisiert und mit Nachdruck ${ }^{151}$ verfolgt wurde:

\footnotetext{
»The general policy of the Ministry was based on the principle of rationing the imports of neutral countries in contact with Germany, combined with purchase schemes for their exports, e.g. we would say to Holland, Denmark, or Norway: >Unless you stop sending supplies to Germany we will not let you have the coal or sugar which is essential to you. But if you will stop sending supplies, or limit them, we will buy them ourselves at a fair price, and in addition we will let you have as much coal or sugar as you can prove you require for your own consumption-always on condition that you don't send it on to Germany $\prec \cdot \ll^{152}$
}

Wirklich »fair « waren die britischen Angebote an die Neutralen freilich nicht. In aller Regel nämlich lagen die »Purchasing Agreements« unter den deutschen Angeboten, entfalteten aber zusammen mit den britischen Druckmitteln ihre Wirkung. ${ }^{153}$ Eine Vereinbarung mit dänischen Fischern über den Export ihrer Fänge nach Großbritannien anstelle von Deutschland beispielsweise wurde vor dem Hintergrund erzielt, dass die Dänen für den Betrieb ihrer Boote auf britische

148 Chatterton, The Big Blockade (wie Anm. 17), S. 29 f.

149 Ebd., S. $237 \mathrm{f}$.

150 Ein entsprechender Act of Parliament erging am 24.6.1915, Cobb, Preparing for Blockade (wie Anm. 2), S. 255.

151 Auch vorherige Kritiker einer zu weichherzigen Blockadepolitik wurden in das Ministerium berufen, Osborne, Britain’s Economic Blockade (wie Anm. 5), S. 121-123; beachte auch Bell, A History of the Blockade (wie Anm. 11), S. 452-454.

152 Hankey, The Supreme Command, vol. 2 (wie Anm. 16), S. $547 \mathrm{f}$.

153 Osborne, Britain's Economic Blockade (wie Anm. 5), S. $132 \mathrm{f}$. 
Öllieferungen angewiesen waren. ${ }^{154}$ Ähnliche Abkommen wurden mit Norwegen, der Schweiz und den Niederlanden getroffen, wodurch beispielsweise deren Rindfleischexport nach Deutschland im Jahr 1917 angeblich vollständig verhindert werden konnte. ${ }^{155}$

Neben diesen eher großflächigen Ansätzen gelang es Großbritannien insbesondere nach der Einrichtung des Ministry of Blockade im Frühjahr 1916, mithilfe des bereits erwähnten Systems schwarzer Listen verstärkt Druck auf einzelne Händler und Schiffseigner auszuüben. ${ }^{156}$ Die vor allem mithilfe der Zensur identifizierten Händler, die Waren für Deutschland oder deutsche Scheinfirmen transportierten, erhielten zunächst einmal keinen Raum mehr auf britischen Schiffen. Zudem wurde ihnen die Versicherung ihrer Waren verweigert, wobei Großbritannien zugutekam, dass der Markt für entsprechende Policen von Lloyd's of London dominiert wurde. Gegenüber Schiffseignern aus neutralen Ländern kam das bereits erläuterte Druckmittel der britischen Kohle hinzu. ${ }^{157}$ Schon im Sommer 1916 zeigte das Vorgehen Wirkung. Selbst Schiffe, die zuvor immer wieder versucht hatten, die Blockade zu umgehen, liefen nun britische Häfen wie Kirkwall an, um die eigene Ladung untersuchen zu lassen. ${ }^{158}$

Ein wesentlicher Schwachpunkt der schwarzen Listen war jedoch, dass die USA entschlossen protestierten und ihre Händler deshalb ausgenommen wurden. Um dieses Problem zu umgehen, entwickelte das britische Foreign Office das Konzept des »Navycert«. ${ }^{159}$ Diese Zertifikate wurden vom März 1916 an bis zum Kriegseintritt der USA im April 1917 an US-amerikanische Firmen vergeben und ermöglichten die Durchfahrt der Blockadelinien ohne weitere Untersuchung. Interessierte Firmen konnten sie bei der britischen Botschaft beantragen. ${ }^{160}$ Diese übermittelte die Anfragen nach London, wo sie in aller Regel innerhalb von zwei Tagen bearbeitet wurden - und in drei von vier Fällen auch genehmigt. Für die beteiligten Firmen bedeutete dieses Verfahren eine erleichterte Passage, während

154 Bell erwähnt einige Versuche, Neutrale unter Druck zu setzen, die daran scheiterten, dass diese andere Quellen für die zuvor von Großbritannien bezogenen Waren fanden, Bell, A History of the Blockade (wie Anm. 11), S. 606.

155 Chatterton, The Big Blockade (wie Anm. 17), S. 257.

156 Chair, The Sea is Strong (wie Anm. 13), S. 219f.; Halpern, World War I (wie Anm. 11), S. 100; Osborne, Britain's Economic Blockade (wie Anm. 5), S. 164.

157 Chatterton, The Big Blockade (wie Anm. 17), S. 256f.; Osborne, Britain’s Economic Blockade (wie Anm. 5), S. 125.

158 Chatterton, The Big Blockade (wie Anm. 17), S. 263.

159 Osborne, Britain's Economic Blockade (wie Anm. 5), S. 125. Für die vorbereitenden Maßnahmen dazu siehe auch Cobb, Preparing for Blockade (wie Anm. 2), S. $256 \mathrm{f}$.

160 Bell, A History of the Blockade (wie Anm. 11), S. 457-459; Osborne, Britain's Economic Blockade (wie Anm. 5), S. $125 \mathrm{f}$. 
Großbritannien eine verbesserte Kontrolle über die Waren gewann, die über den Ozean transportiert wurden, bevor die Schiffe überhaupt in See stachen. ${ }^{161}$

Es war schließlich der uneingeschränkte U-Boot-Krieg, den Deutschland im Februar 1917 erklärte, der diesem Prinzip der Vorabkontrolle neutraler Handelsschiffe nachhaltig zur Durchsetzung verhalf. Die Furcht vor den Großbritannien »belagernden« U-Booten nämlich führte dazu, dass Reeder und Kapitäne neutraler Schiffe das Risiko, auf See gestoppt oder in britische Häfen umgeleitet zu werden, für zu groß hielten. Viele Schiffe blieben deshalb im Februar in ihren Häfen. ${ }^{162}$ Um diesem Problem für den eigenen Handel entgegenzutreten, bot Großbritannien ab Mitte des Monats an, neutrale Handelsschiffe außerhalb der europäischen Gewässer zu kontrollieren und mit »Green Cards « zu versehen, die ihnen die unbehelligte Passage durch die Blockadelinien ermöglichen sollten - während Schiffe, die nicht vorab untersucht worden waren und neutrale Nachbarländer Deutschlands ansteuerten, unter den Generalverdacht des Konterbandetransports gestellt wurden; sie sollten fortan als Prisen beschlagnahmt werden dürfen. ${ }^{163}$

Schon im März zeigte das neue britische Verfahren Wirkung: Die neutralen Händler griffen auf die vorverlagerten Untersuchungen zurück und steuerten wieder nordeuropäische Gewässer an, ${ }^{164}$ nachdem sie im kanadischen Halifax, in Kingston auf Jamaica, im ägyptischen Port Said oder Freetown in Sierra Leone kontrolliert worden waren. ${ }^{165}$ Die Kombination aus Navycerts und Green Cards reduzierte die Rolle der Blockadeflotte also von der des entscheidenden Kontrolleurs auf diejenige eines Druckmittels neben der Rationierung, dem Zugang zu Kohle, Öl und anderen Rohstoffen - und dem deutschen U-Boot-Krieg.

Vervollständigt wurde diese Drohkulisse schließlich durch den Kriegseintritt der USA im April 1917. Zwar musste Großbritannien noch im Mai US-Firmen aus den schwarzen Listen entfernen, da die US-Regierung sonst nicht bereit gewesen wäre, das System mitzutragen. Auch etablierten die USA zunächst ein eigenes Embargosystem, unabhängig von der britischen Blockademaschinerie. Nach und nach jedoch verschärfte die US-Regierung die Regeln für den Export heimischer Waren und verlieh dem Druckmittel der Rationierung eine nachdrückliche Wirkung. ${ }^{166}$ Chatterton bringt die Situation nach dem US-Kriegseintritt auf den Punkt:

161 Chatterton, The Big Blockade (wie Anm. 17), S. 256.

162 Osborne, Britain's Economic Blockade (wie Anm. 5), S. $154 \mathrm{f}$.

163 Tagebucheintrag Fitzroy, 16.2.1917, zit. nach Fitzroy, Memoirs (wie Anm. 80), S. 643f.; Chatterton, The Big Blockade (wie Anm. 17), S. 274-276.

164 Osborne, Britain's Economic Blockade (wie Anm. 5), S. $154 \mathrm{f}$.

165 Chatterton, The Big Blockade (wie Anm. 17), S. 275.

166 Osborne, Britain's Economic Blockade (wie Anm. 5), S. 162f. und S. 173; siehe auch Hawkins, The Starvation Blockades (wie Anm. 33), S. 193-198. 
»[T]here was virtually no need for a Blockade Fleet to intercept and examine suspected cargoes-the latter never reached the holds! It was not the 10th Cruiser Squadron with its boarding parties who now did the stoppage, but the Customs Officers in New York, Boston, Norfolk (Virginia), and other Atlantic ports. $\aleph^{167}$

Gemeinsam dominierten Frankreich, Großbritannien und die USA den Weltmarkt für den Großteil der (kriegs-)wichtigen Güter. Neutrale Staaten konnten es sich nicht leisten, gegen die Spielregeln dieser drei Nationen zu verstoßen. ${ }^{168}$ Der Einsatz eines Blockadegeschwaders als Druckmittel war damit überflüssig geworden. Sein Ende zeichnete sich daher schon deutlich vor der offiziellen Auflösung ab. Noch einmal Chatterton:

»[B]y the end of October [1917] the Squadron as a Blockading force came to an end: the people in offices ashore, with their typewriters, telegraphs and files, their network of agreements, trading trusts, and treaties, were carrying on the operation as if it were some worldextensive business with branches on both sides of the Atlantic. It was no longer a seaman's job. «169

\section{Blockade ohne Blockade - Der Weltkrieg als Katalysator}

Die Blockade Deutschlands war im Verlauf des Ersten Weltkriegs nicht allein ausgeweitet und verschärft, auch ihre Durchsetzung war grundlegend reformiert worden. Beide Veränderungen erfolgten schrittweise und als Reaktion auf die Kriegserfahrungen einerseits beziehungsweise vor dem Hintergrund der Eskalation des Konfliktes und gegnerischer Handlungen andererseits. Insbesondere der Umstand, dass die Blockade zunehmend mit bürokratischen Mitteln in von Europa weit entfernten Häfen durchgesetzt werden konnte, verdankt sich letztlich dem Umstand, dass das britische Foreign Office die Blockade nur langsam verschärfte und dadurch die Kooperationsbereitschaft der USA stets aufrecht erhielt. Seine Kritiker, die wie Admiral Jellicoe immer wieder klagten, bei der Ausgestaltung der Blockade würden zu viele Rücksichten auf die Diplomatie genommen, das Foreign Office sei »imbecile« und "afraid of their own

167 Chatterton, The Big Blockade (wie Anm. 17), S. 277.

168 Hawkins, The Starvation Blockades (wie Anm. 33), S. 193-198; Chatterton, The Big Blockade (wie Anm. 17), S. 276f.; Osborne, Britain's Economic Blockade (wie Anm. 5), S. 173.

169 Chatterton, The Big Blockade (wie Anm. 17), S. 279; beachte auch Tupper, Reminiscences (wie Anm. 14), S. 264. 
shadows ${ }^{170}$, waren letztlich im Irrtum. ${ }^{171}$ Denn erst mit dem Kriegseintritt der USA wurde die Blockade wirklich effektiv. ${ }^{172}$ Zugleich hatte sie nur noch wenig mit der Blockade gemein, die zu Kriegsbeginn von Großbritannien verhängt worden war. Aus einer durch eine Seemacht mit Kriegsschiffen durchgesetzten Handelssperre für eine eng begrenzte Liste kriegsbezogener Güter war eine umfangreiche Blockade von Waren geworden, die der gegnerischen Nation potenziell nützlich sein könnten, und die nicht mehr auf See abgefangen, sondern deren Handel mit dem Gegner mit einer Mischung aus politischen Anreizen und Druckmitteln unterbunden wurde, auch bei dessen neutralen Nachbarstaaten. ${ }^{173}$ Die neue Blockade, die ohne ein Blockadegeschwader aufrechterhalten wurde, war nicht länger eine »rein« militärische Maßnahme, sondern ein politisch-diplomatisches Machtmittel. Als solches blieb sie während der Waffenstillstandsverhandlungen mit Deutschland in Kraft und sollte Widerstände gegen die alliierten Bedingungen im Keim ersticken. ${ }^{174}$ Sie wurde erst aufgehoben, als der Friedensvertrag ratifiziert wurde. ${ }^{175}$ Dass ihre neue Form auch zukünftig Anwendung finden würde, schien bereits Hankey klar: »Blockade is one of the questions which has been altered by the developments in this war, and the law governing it will certainly have to be altered. There is no danger, however, that it will be abolished. ${ }^{176}$ Ganz im Gegenteil: Durch den totaler werdenden Ersten Weltkrieg war aus ihr ein Werkzeug geworden, das im 20. Jahrhundert in unterschiedlichsten Situationen angewendet wurde und nach wie vor angewendet wird - nicht selten mit dem Ziel, internationale Konflikte ohne die Anwendung von Waffengewalt zu entscheiden.

170 Jellicoe an Fisher, 20.1.1916, zit. nach 1893-1916 (wie Anm. 69), S. 201; für weitere Beispiele siehe Chatterton, The Big Blockade (wie Anm. 17), S. 66; 1893-1916 (wie Anm. 69), S. 139.

171 Beachte hierzu auch Cobb, Preparing for Blockade (wie Anm. 2), S. 255; ferner Chatterton, The Big Blockade (wie Anm. 17), S. $237 \mathrm{f}$.

172 Siehe oben, Anm. 71.

173 Beachte auch Erich Raeder, Das Kreuzergeschwader, Berlin 1922 (= Der Kreuzerkrieg in den ausländischen Gewässern, 1), S. $40 \mathrm{f}$.

174 Osborne, Britain’s Economic Blockade (wie Anm. 5), S. 181f.; Schreiben Ernest M. Pollock mit Memorandum zum Trade War vom 27.6.1917, zit. nach British Documents on Foreign Affairs. Reports and Papers from the Foreign Office Confidential Print, p. 2: From the First to the Second World War. Serie H: The First World War, 1914-1918, vol. 8: Blockade and Economic Warfare, p. 4: November 1916-November 1918. Ed. by David Stevenson, Frederick 1989, S. 210-220; TNA, Cab 23/8, Memorandum des War Cabinet vom 11.10.1918, S. 10; siehe ferner oben, Anm. 10.

175 Bell, A History of the Blockade (wie Anm. 11), S. 709; Osborne nennt den 12.7.1919, Osborne, Britain's Economic Blockade (wie Anm. 5), S. 189.

176 Hankey, The Supreme Command, vol. 2 (wie Anm. 16), S. 862. 